\title{
Liste der im Handbuch angewandten Literatur-Abkürzungen.
}

\section{Zeitschriften.}

\begin{abstract}
American Chemical Journal
\end{abstract}
American Journal of Science (Silliman)

Annslen der Chemie (Liebig)

Annalen der Physik und Chemie (Poggendorff)

(Witdemann)

Annalen der Physik "
Abkirxungen

Am. Oh. J.

Am. J. Science

Ann.

Pogg.

Wied.

Ann. Phys.

Ann. chim. phys.

Beibl.

Ber.

Ber. Phys. Ges.

Bull.

Chem. N.

Ch. $Z$.

Chemische Industrie, Die . . . . . . . . . . . . . . . Ch. Ind.

Chemisches Zentralblatt . . . . . . . . . . . . . . Zentr.

Chemische Zeitschrift . . . . . . . . . . . . . . . Ch.Ztschr.

Comptes rendus de l'Academie des Sciences (Paris) . . . . . . C. r.

Dinglers Polytechnisches Journal . . . . . . . . . . . . Dingl.

Elektrochemische Zeitschrift . . . . . . . . . . . . . . Elektr. Ztschr.

Gazzetta chimica italiana . . . . . . . . . . . . . . Gazz.

Jahresbericht über die Fortschritte der Chemie etc. . . . . . Jahr.

Journal de Chimie physique . . . . . . . . . . . . . J. Chim. phys.

Journal de Physique . . . . . . . . . . . . . . . . . J. phye.

Journal für praktische Chemie . . . . . . . . . . . . J. pr. Ch.

Journal of Physical Chemistry . . . . . . . . . . . . . J. Physic. Chem.

Journal of the American Chemical Society . . . . . . . . . J. Au. Ch. S.

Journal of the Chemical Society (London) . . . . . . . . . J. Chem. S.

Journal of the Society of Chemical Industry . . . . . . . . J. Soc. Cb. Ind.

Mitteilungen aus dem Kgl. Materialprüungsamt zn Groblichterfelde Mitt. Mater. Amt

Monatshefte für Chemie . . . . . . . . . . . . . . . . Mfte.

Moniteur scientifique . . . . . . . . . . . . . . . . Monit.

Österreichische Chemiker-Zeitung . . . . . . . . . . . . Öst. Ch. Ztg.

Pharmaceutical Journal Transactions . . . . . . . . . . . Pharm. J. Trans.

Pharmazeutische Zeitung . . . . . . . . . . . . . Pharm.Z.

Pharmazentische Zentralhalle . . . . . . . . . . . . Pharm. Zentr. 
Liste der im Handbuch angewandten Abkürzungen.

\section{Ablürxungen}

Philosophical Magazine . . . . . . . . . Phil. Mag.

Physikalische Zeitschrift . . . . . . . . . . . . . . Phys. Ztsehr.

Proceedings of the Chemical Society (London) . . . . . . . Proc. Chem. S.

Recueil des travaux chimiques des Pays-Bas . . . . . . . . Rec. trav. chim. .

Sitzungsberichte der Kgl. Akademie der Wissenschaften, Wien . . Sitggb. Wien.

Sitzungabericbte der Kgl. PreuBischen Akudemie der Wise enschaften,

Berlin .

Sitzungsberichte der Kgl. Bayrischen Akademie der Wissenschaften, mathemat.-naturwissenschaftliche Abteilung

Sitzgb. Berlin

Sitzgb. Bayr.

Stahl and Eisen. . . . . . . . . . . . . . . . . St. E.

Zeltsehrift für analytiache Chemie . . . . . . . . . . . Z. ansl. Ch.

Zeitschrift für ungewandte Chemie . . . . . . . . . . . . Z. angew. Ch.

Zeitschrift für anorganische Chemie . . . . . . . . . . . . Z. anorg. Ch.

Zeitschrift für Chemie und Industrie der Kolloide . . . . . . Z. Kolloid.

Zeitachrift für chemiache Apparatenkunde . . . . . . . . . Z. Appar.

Zeitschrift für physikaliachen und chemischen Unterricht. . . . Z. Unterr.

Zeitschrift fír Elektrochemio . . . . . . . . . . . . . . Z. Elektr.

Zeitschrift für Instrumentenkunde . . . . . . . . . . . . Z. Instr.

Zeitschrift für physikalische Chemie . . . . . . . . . . Z. phys. Ch.

\section{Handbücher.}

Abderhalden, Handbuch der biochemischen Arbeitsmethoden

Abegg-Auerbach, Handbuch der anorganischen Chemie

Arendt-Doermer, Technik der anorganischen Experimentalchemie. 1910 . . . . . . . . . .

Bender-Erdmann, Chemiache Präparatenkunde. Zrei Bände. 1893 . . . . . . . . . . . . .

Berzelius, Lehrbuch der Chemie. 4. Aufl. Zehn Bände. $1885-1841$. . . . . . . . . . .

Dammer, Handbuch der anorganischen Chemie . .

Fehling, Handworterbuch der Chemie. . . . . .

Gmelin-Kraut, Handbuch der anorganischen Chemie. 6. Auflago . . . . . . . . . . . . . .

Gmelin-Kraut, Handbuch der anorganischen Chemie. 7. Aut lage, heranggegeben von Friedheim u. Peters

Graham-Otto, Ausfuhrliches Lehrbuch der Chemie. 5. Auflage von Michaelis. . . . . . . . .

Landolt-Börnstein, Phyoik.-chem. Tabellen. 3. Aufl. 1905

desgl. 4. Aufl. 1912 . . . . . . . . . . . . . .

Moissan, Traité de chimie minérale. 1906 . . . .

Muspratt, Handbuch der technischen Chemie. 1891 .

Ontwrld-Luther, Physikochemische Messungen. 3. Aufl. 1910 . . . . . . . . . . . . . . . .

Roscoø-Schorlemmer, Ausfubrliches Lebrbuch der Chemie . . . . . . . . . . . . . . .

Travers-Estreicher, Experimentelle Untersuchung von Gasen. 1908

\section{Abkilrxungen}

Abderhalden

Abegg-Auerbach

Arendt-Doermer

Bender-Erdmann

Berzelius

Dammer

Fehling

Gmelin-Kraut

Grmelin-Kraut-Friedheim-Peterrs

Graham-Otto-Michaelig

Landolt-Börnstein

Landoit-Börnstein-Roth

Moisagn

Muspratt

Ostwald-Luther

Roscoe-Schorlemmer

Travers-Estreicher 